\title{
Crisis económica española y salud. Impacto clínico en el dolor crónico
}

\author{
M. Cortiñas-Sáenz ${ }^{1}$, J.L. Bonilla-García ${ }^{1}$, C. Arnedo Ruiz ${ }^{1}$, A.I. Navajas Gómez de Aranda ${ }^{1}$, \\ M.B. Menoyo Alonso ${ }^{2}$, R. López Martín ${ }^{1}$, M.I. Carricondo Martínez ${ }^{3}$ y F. Jerez Collado ${ }^{3}$ \\ ${ }^{1}$ Servicio de Anestesiología y Reanimación. Hospital Torrecárdenas. Almería. ${ }^{2}$ Hospital de Basurto. Bilbao. \\ ${ }^{3}$ Unidad de Dolor Crónico. Hospital Torrecárdenas. Almería.
}

Cortiñas-Sáenz M, Bonilla-García JL, Arnedo Ruiz C, Navajas Gómez de Aranda AI, Menoyo Alonso MB, López Martín R, Carricondo Martínez MI, Jerez Collado F. Crisis económica española y salud. Impacto clínico en el dolor crónico. Rev Soc Esp Dolor 2015; 22(2): 51-58.

\footnotetext{
ABSTRACT

Background: Many European governments have abundantly cut down public expenditure on health during the financial crisis. Consequences of the financial downturn on health outcomes have begun to emerge.

The recession has also driven structural reforms, and affected the priority given to public policies. The purpose of this paper is to analyse how austerity impacts health in Spain and better understand the response of European health systems to the financial crisis.

Material and methods: Prospective study of 206 patients over a period of two years. Was evaluated various pharmacological and invasive treatments and medical outcomes in Chronic Pain Unit depending on the patient's income level.

Results: The economic situation of the patient is statistically significantly correlated with the success of medical treatment ( $p$ $=0.01$ ) and the occurrence of various adverse effects in relation to medical treatment $(p<0.01)$. Results provide strong evidence of a statistically significant negative effect of the financial crisis on health trends.

Conclusions: Policy decisions about how to respond to economic crises have pronounced and unintended effects on public health. The governance and leadership find ways to mitigate its effects.
}

Key words: Health policy. Public health. Health status. Financial crisis. Economic crisis. Pain.

\section{RESUMEN}

Antecedentes: múltiples gobiernos europeos han reducido de forma considerable el gasto público en salud durante la crisis económica. Las posibles consecuencias de la crisis financiera sobre los resultados en la salud han comenzado a aparecer.

La recesión también ha impulsado diversas reformas estructurales que han afectado a la prioridad otorgada a las políticas públicas. El propósito de este trabajo es analizar el posible impacto de las políticas de austeridad en la salud en España, y así entender la respuesta de los sistemas sanitarios europeos a la crisis financiera y políticas de austeridad.

Material y métodos: estudio prospectivo de 206 pacientes en una Unidad de Dolor Crónico durante un periodo de dos años. Se evaluaron los tratamientos farmacológicos, invasivos y los resultados médicos de la unidad en función del nivel de ingresos del paciente.

Resultados: la situación económica del paciente se correlacionó de forma estadísticamente significativa con el éxito del tratamiento médico $(p=0,01)$, así como en la aparición de diversos efectos adversos en relación al tratamiento médico ( $p$ $<0,01)$. Existe una fuerte evidencia de un efecto negativo estadísticamente significativo de la actual crisis financiera sobre los resultados de sanitarios.

Conclusiones: las decisiones políticas y su respuesta a las crisis económicas han acentuado los efectos no deseados sobre la salud pública. El gobierno y los diversos líderes deben hallar fórmulas eficientes para mitigar sus efectos.

Palabras clave: Política sanitaria. Salud pública. Estado de salud. Crisis económica. Crisis financiera. Dolor. 


\section{INTRODUCCIÓN}

La crisis económica y financiera de 2008 se enmarca en un contexto a nivel mundial y es considerada como una «crisis de los países desarrollados». El deterioro de los principales indicadores macro-económicos se ha extendido hasta los planos político, social y financiero. Esta crisis ha afectado de una manera dramática a los países del sur de Europa, en donde las medidas impuestas ante esta situación han sido básicamente políticas de austeridad y contención del gasto público $(1,2)$. Existen discrepancias entre diversos autores si estas políticas sanitarias han generado un aumento de malos estados de salud, enfermedades transmisibles, tasas de ansiedad, depresión y suicidios en personas económicamente vulnerables $(1,2,4)$.

La crisis económica incide en los recursos asignados al sistema sanitario público los cuales son necesarios para garantizar un nivel óptimo de equidad, de prestaciones y calidad asistencial $(2,5)$. Los sistemas sanitarios son un recurso de vital importancia para contener los efectos de la transformación de la crisis económica en crisis social, razón por lo que debería preservarse de acciones dirigidas a disminuir el volumen de recursos asignados, ya que podría incidir en una dificultad en el acceso a una sanidad de calidad de diversos colectivos.

Las crisis económicas pueden tener efectos positivos sobre la salud si existe un estado de bienestar que garantice las necesidades mínimas, ya que pueden conllevar una mejora de los estilos de vida, niveles educativos y salud medioambiental (6).

El dolor tiene un impacto directo en la calidad de vida del paciente, su ámbito social y familiar, conllevando graves repercusiones económicas y laborales. La importancia de dolor se constata al ser considerada por la Organización Mundial de la Salud como el quinto signo vital. El Plan Andaluz de Atención a las Personas con Dolor (7) revela que el dolor crónico es el causante del 50\% de todas las bajas laborales y pudiera asociarse a una baja productividad. Dicho plan enfatiza en la atención sanitaria precoz en los cuadros de dolor crónico puesto que los costes asociados a él suponen un gasto superior a los 3.000 millones de euros anuales (2,5\% del PIB de nuestro país).

El objetivo del presente trabajo es evaluar el posible impacto de la actual crisis económica en el tratamiento y resultados médicos de pacientes con dolor crónico de diversa etiología en función de la renta del paciente.

\section{MATERIAL Y MÉTODOS}

Estudio prospectivo y observacional de una cohorte de pacientes con dolor crónico. El periodo de estudio abarca los años 2011 y 2012.

\section{Pacientes y métodos}

Los criterios de inclusión fueron todos los pacientes con dolor crónico de cualquier etiología (Tabla I), remitidos desde atención especializada y/o primaria a una Unidad de Dolor Crónico y atendidos por el mismo médico anestesiólogo. El único criterio de exclusión fue la ausencia de consentimiento verbal del paciente a participar en el estudio. En la figura 1 se expone el diagrama de flujo de pacientes.

\section{Consideraciones técnicas}

Tras firmar consentimiento informado para participar en el estudio y tras la entrevista médica, se ofertaron las opciones médicas y/o las técnicas invasivas o bloqueos en función de la patología de base y comorbilidad del enfermo. Los diferentes tipos de bloqueos en función de la situación económica del paciente se exponen en la tabla II. El protocolo de la unidad indica que en todos los casos se realizaba un bloqueo diagnóstico con anestésicos locales y/o esteroides; y si este conllevaba una mejoría superior al $50 \%$ en una escala visual analógica (EVA) con una duración temporal de varios meses, se repetía el procedimiento al reaparecer el dolor. En aquellos casos con una reducción del dolor superior al 50\% y una duración temporal muy limitada se realizó bloqueo con toxina botulínica, agentes neurolíticos o termocoagulación con radiofrecuencia sobre la estructura originaria del cuadro álgico.

\section{Variables}

Se recogieron diversas variables demográficas, situación económica de toda la unidad familiar (buena: ingresos anuales superiores a $40.000 €$; normal: ingresos económicos entre 12.000 y $40.000 €$; mala: ingresos económicos inferiores a $12.000 €$ ) antes del inicio del periodo de crisis y en el momento del estudio, situación laboral (activo, parado con prestación, parado sin prestación, pensionista), número de comorbilidades médicas asociadas, existencia de tratamiento médico y/o invasivo, número de fármacos por pacientes, empleo de novedades terapéuticas, tipo de técnica intervencionista realizada, tiempo de inicio del cuadro álgico, intensidad del dolor mediante una EVA de 11 puntos de 0 a 10 ( 0 sin dolor y 10 definido como el peor dolor imaginable), etiología, topografía y características del dolor. Se evaluó la tasa de éxito del tratamiento médico y/o invasivo si existía una disminución de al menos un 50\% en la escala EVA, del grado de satisfacción de la técnica anestésica (тиу satisfecho, satisfecho, poco satisfecho y nada satisfecho). Posteriormente se realizó entrevista telefónica y/o revi- 
TABLA I. ETIOLOGÍA DE LOS CUADROS DE DOLOR Y SU RELACIÓN CON TRATAMIENTOS MÉDICOS E INVASIVOS ADMINISTRADOS (UN MISMO PACIENTE PUEDE RECIBIR AMBOS TIPOS DE TRATAMIENTOS) DURANTE EL PERIODO DE ESTUDIO Y SEGUIMIENTO

\begin{tabular}{|c|c|c|c|c|}
\hline Etiología & $N .^{o}$ & $\%$ & Trat. médico & Trat. invasivo \\
\hline Síndrome postlaminectomía & 44 & 21,35 & 44 & 26 \\
\hline Estenosis de canal & 19 & 9,23 & 16 & 11 \\
\hline Oncológico & 18 & 8,73 & 18 & 2 \\
\hline Raquialgia mecánica & 17 & 8,25 & 14 & 2 \\
\hline Gonartrosis & 16 & 7,76 & 14 & 9 \\
\hline Síndrome miofascial & 15 & 7,28 & 8 & 11 \\
\hline Fibromialgía & 12 & 5,82 & 10 & 0 \\
\hline Totalgía & 12 & 5,82 & 8 & 0 \\
\hline Hombro doloroso: & 11 & 5,33 & 11 & 7 \\
\hline Artrósica & 6 & $(2,91)$ & 6 & 4 \\
\hline Tendinosa & 5 & $(2,42)$ & 5 & 3 \\
\hline Coxartrosis & 9 & 4,36 & 8 & 3 \\
\hline Úlceras vasculares & 7 & 3,39 & 7 & 1 \\
\hline Neuropatía diabética & 7 & 3,39 & 7 & 0 \\
\hline Síndrome regional complejo & 6 & 2,91 & 6 & 2 \\
\hline Neuralgias faciales & 5 & 2,42 & 5 & 2 \\
\hline Necrosis avascular de cadera & 4 & 1,94 & 4 & \\
\hline Cefaleas & 2 & 0,97 & 2 & 1 \\
\hline Síndrome miembro fantasma & 2 & 0,97 & 2 & 0 \\
\hline Total & 206 & 100 & 184 & 77 \\
\hline
\end{tabular}

N. ${ }^{\circ}$ : número de casos.\%: porcentaje de casos. Trat. médico: número de pacientes de dicha patología tratados con fármacos. Trat. invasivo: número de pacientes de dicha patología tratados mediante técnicas invasivas.

sión de historia clínica para evaluar la evolución a 1, 3 y 6 meses postratamiento.

\section{Aspectos éticos}

El presente trabajo respeta los principios fundamentales establecidos en la declaración de Helsinki, así como los establecidos en el convenio del consejo de Europa relativos a los derechos humanos y la biomedicina, y los establecidos en la legislación española en el campo de la bioética. El tratamiento, la comunicación y la cesión de los datos de carácter personal de todos los sujetos participantes se ajustó a lo dispuesto en la Ley Orgánica 15/1999, de 13 de diciembre, de protección de datos de carácter personal. Sólo se transmiten los datos recogidos para el estudio que en ningún caso contendrán información que pueda identificar al paciente directamente.

\section{Análisis estadístico}

El análisis estadístico se realizó con el programa Stata $^{\circledR} 7$ (Stata Corporation, Computing Resource Center, College Station, Texas. EE. UU.). Se realizó un estudio descriptivo de las variables y se calculó la distribución de frecuencias de estas de forma global. Se testó la distribución normal mediante el test de Kolomogorov-Smirnov, y se comparó la diferente distribución del éxito y fracaso de los diversos tratamientos en los pacientes de la serie en función de la situación económica entre las variables recogidas y su posible asociación utilizando para las variables cuantitativas el test de la "t" de Student o la prueba de Kruskall-Wallis, si no existía igualdad de varianzas, y para las variables cualitativas el test de la Chi-cuadrado $\left(\mathrm{Ji}^{2}\right)$ con corrección de Yates o el test de Fischer. Se admitió un nivel de significación de $\mathrm{p}<0,05$ para todos los estadísticos utilizados. 


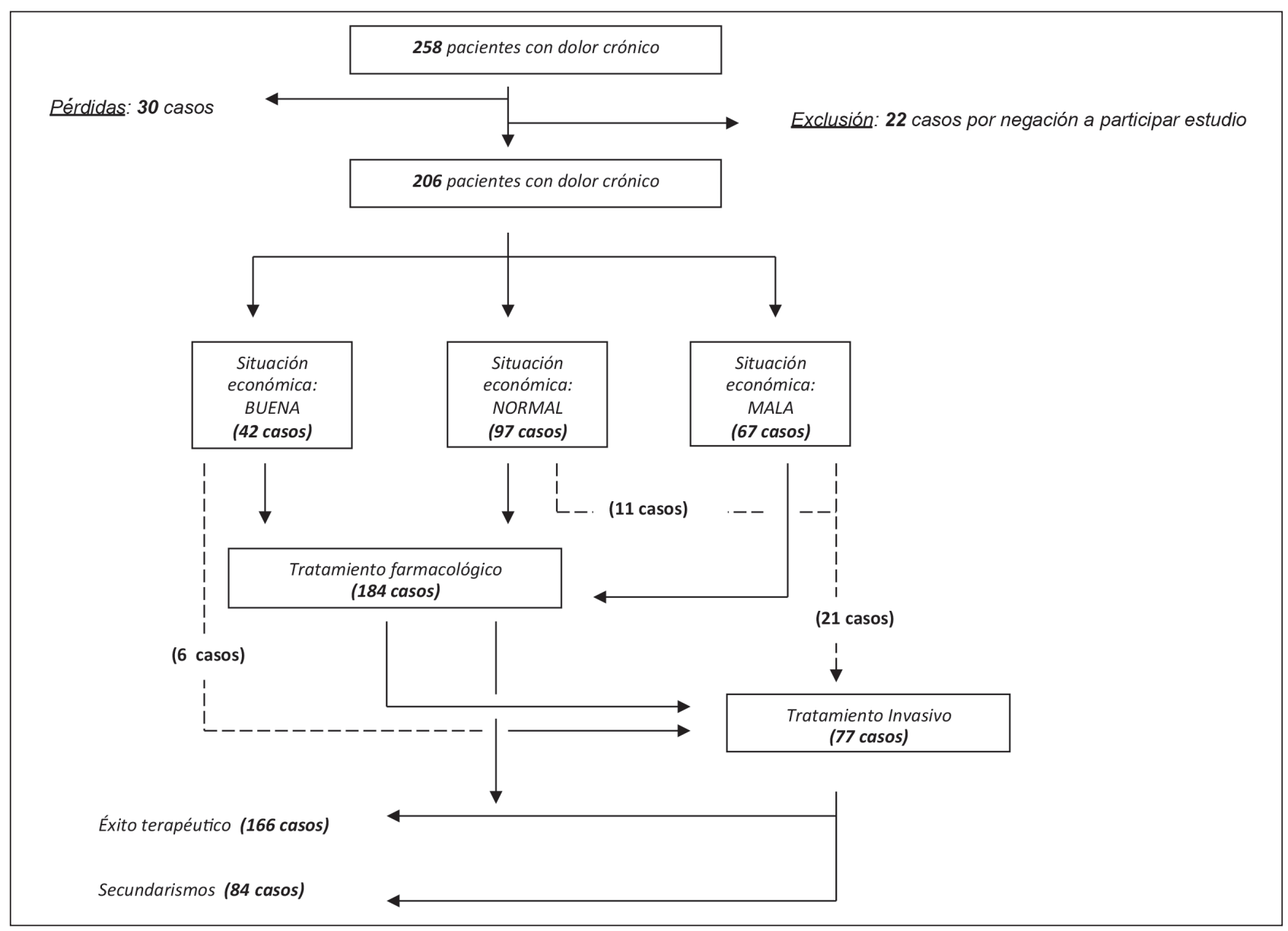

Fig. 1. Diagrama de flujo. Las líneas discontinuas indican aquellos casos en los que en la primera consulta fueron remitidos directamente para la realización de técnicas invasivas.

\section{RESULTADOS}

De los 258 pacientes incluidos en el estudio, 22 fueron excluidos por negación a participar en el mismo (Fig. 1). Durante los 6 meses de seguimiento se produjeron 30 pérdidas por imposibilidad de contacto telefónico. La causa más frecuente de consulta de dolor crónico en nuestra cohorte fue el síndrome postlaminectomía o cirugía fallida de espalda (Tabla I).

Un 32,52\% de la cohorte presentaban una mala situación económica, principalmente en los sectores de parados con o sin prestaciones, población inmigrante y con un bajo nivel de estudios. Un 85,05\% de los pensionistas de nuestro estudio presentan una situación financiera considerada buena o normal. La precariedad económica se correlacionó de forma significativa con una dificultad en la adquisición del tratamiento médico, una mayor incidencia de trastornos en la esfera mental, una menor utilización de medicamentos tipo novedades terapéuticas y del número de analgésicos de forma global por paciente.
La situación económica del paciente se correlacionó de forma estadísticamente significativa con el éxito del tratamiento médico $(\mathrm{p}=0,01)$, así como en la aparición de diversos efectos adversos en relación al tratamiento médico $(\mathrm{p}<0,01)$. Las diferencias estadísticamente significativas entre las distintas variables recogidas en nuestro estudio se exponen en la tabla III. Las medidas terapéuticas intervencionistas son la primera opción de tratamiento en un mayor número de pacientes con peor situación económica.

La tabla IV y figura 2 exponen la actividad asistencial y evolución de la lista de espera médica y quirúrgica de la unidad a lo largo del periodo de estudio.

\section{DISCUSIÓN}

Existe cierta discrepancia si en el contexto de crisis económica actual, donde las diferentes partidas presupuestarias se reducen de una manera progresiva, las diferentes 
TABLA II. NÚMERO DE CASOS, MEDIA CON DESVIACIÓN ESTÁNDAR (M \pm DE) Y GRADO DE SIGNIFICACIÓN ESTADÍSTICA DE DIVERSAS VARIABLES EN FUNCIÓN DE LA SITUACIÓN ECONÓMICA DEL USUARIO

\begin{tabular}{|c|c|c|c|c|}
\hline \multirow{2}{*}{ Variable } & \multicolumn{3}{|c|}{ Situación económica } & \multirow{2}{*}{ Valor $p$} \\
\hline & Buena & Normal & Mala & \\
\hline \multicolumn{5}{|l|}{ Número pacientes: } \\
\hline Año 2007 & 58 & 113 & 35 & $<0,01$ \\
\hline Año 2011-12 & 42 & 97 & 67 & \\
\hline Edad (años) $\mathrm{M} \pm \mathrm{DE}$ & $48,3 \pm 14,2$ & $69,8 \pm 21,1$ & $51,4 \pm 15,7$ & $<0,01$ \\
\hline Sexo (V/M). & $27 / 15$ & $46 / 51$ & $22 / 45$ & 0,15 \\
\hline \multicolumn{5}{|l|}{ Nivel de estudios: } \\
\hline Sin estudios & 1 & 5 & 25 & \multirow{4}{*}{$<0,01$} \\
\hline Primaria & 2 & 12 & 21 & \\
\hline Secundaria & 16 & 45 & 13 & \\
\hline Universitarios & 23 & 35 & 8 & \\
\hline N. ${ }^{\circ}$ comorbilidad $\mathrm{M} \pm \mathrm{DE}$ & $2,3 \pm 1,5$ & $3,4 \pm 1,9$ & $2,1 \pm 1,6$ & $<0,01$ \\
\hline EVA basal $\mathrm{M} \pm \mathrm{DE}$ & $6,1 \pm 2,2$ & $6,0 \pm 2,4$ & $6,3 \pm 2,3$ & 0,89 \\
\hline Trastorno depresivo & 14 & 26 & 38 & 0,03 \\
\hline Inmigrantes & 5 & 11 & 17 & 0,10 \\
\hline Pensionistas & 12 & 62 & 13 & $<0,01$ \\
\hline \multicolumn{5}{|l|}{ Parados: } \\
\hline Con prestación & 3 & 9 & 19 & \multirow[t]{2}{*}{$<0,01$} \\
\hline Sin prestación & 0 & 2 & 39 & \\
\hline Tratamiento médico & 41 & 92 & 51 & $<0,01$ \\
\hline Número fármacos $\mathrm{M} \pm \mathrm{DE}$ & $4,1 \pm 1,7$ & $4,2 \pm 1,9$ & $2,6 \pm 1.2$ & $<0,01$ \\
\hline Uso novedades terapéuticas & 34 & 51 & 12 & \multirow{5}{*}{$<0,01$} \\
\hline COX II & 8 & 18 & 4 & \\
\hline Tapentadol & 6 & 11 & 2 & \\
\hline Oxicodona-naloxona & 13 & 19 & 4 & \\
\hline Fentanilo* & 7 & 3 & 2 & \\
\hline Dificultad pago fármacos & 0 & 6 & 21 & $<0,01$ \\
\hline Abandono del tratamiento & 5 & 10 & 10 & 0,67 \\
\hline Tratamiento invasivo & 13 & 32 & 32 & 0,09 \\
\hline Éxito tratamiento & 36 & 84 & 46 & 0,01 \\
\hline Secundarismos & 19 & 24 & 34 & $<0,01$ \\
\hline
\end{tabular}

COX II: fármacos inhibidores de la ciclooxigenasa tipo II. Fentanilo*: diversos tipos de fentanilo vía nasal, transmucoso o sublingual para tratamiento del dolor irruptivo.

políticas sanitarias pudieran asociarse a un impacto de los pacientes afectos de ciertas patologías médicas (2). En sentido positivo, un estudio de Regidor y cols. (8) nos muestra que durante el actual periodo de crisis en España, diversos indicadores sanitarios han mejorado de forma paulatina. En sentido contrario, autores como Sydenstricker (9) indican que durante la Gran Depresión se produjeron efectos directos e indirectos sobre la salud de la población a corto y a largo plazo.

El principal hallazgo de nuestro estudio es que una merma de los recursos destinados a la sanidad pública debido a una situación de crisis económica mantenida en el tiempo se asocia a una serie de limitaciones en la actividad asistencial, provoca importantes desigualdades y de resultados 
TABLA III. NÚMERO DE CASOS DE TÉCNICAS INVASIVAS EN FUNCIÓN DE LA SITUACIÓN ECONÓMICA DEL USUARIO

\begin{tabular}{|c|c|c|c|c|}
\hline \multirow{2}{*}{ Variable } & \multicolumn{3}{|c|}{ Situación económica } & \multirow{2}{*}{$\begin{array}{c}N .^{o} \mathrm{C} / \\
\text { Valor } p^{*}\end{array}$} \\
\hline & Buena & Normal & Mala & \\
\hline Número pacientes & 42 & 97 & 67 & 206 \\
\hline \multicolumn{5}{|c|}{ Tipo tratamiento invasivo: } \\
\hline Bloqueo epidural & 3 & 5 & 4 & 12 \\
\hline Intrarticulares & 1 & 2 & 3 & 6 \\
\hline Supraescapular & 1 & 2 & 1 & 4 \\
\hline Geniculados & 0 & 3 & 2 & 5 \\
\hline Femoral y obturador & 0 & 1 & 2 & 3 \\
\hline Trigémino & 0 & 1 & 2 & 3 \\
\hline Bloqueo estrellado & 1 & 2 & 1 & 4 \\
\hline Bloqueo impar & 1 & 3 & 3 & 7 \\
\hline Bloqueo caudal & 2 & 3 & 3 & 8 \\
\hline Bloqueo facetario & 0 & 2 & 3 & 5 \\
\hline Ganglio raíz dorsal & 1 & 2 & 2 & 5 \\
\hline Miofascial & 1 & 3 & 3 & 7 \\
\hline Otros & 2 & 3 & 3 & 8 \\
\hline Tratamiento invasivo & 13 & 32 & 32 & $0,09 *$ \\
\hline
\end{tabular}

N. ${ }^{\circ}$ : número de casos. El bloqueo miofascial incluye la musculatura piriforme, cuadrado lumbar y psoas.

TABLA IV. ACTIVIDAD ASISTENCIAL EN UNIDAD DE DOLOR POR SEMESTRES DE ESTUDIO

\begin{tabular}{lcccc}
\hline & \multicolumn{4}{c}{ Semestre $y$ año } \\
\cline { 2 - 4 } & $1 S-2011$ & $2 S-2011$ & $1 S-2012$ & $2 S-2012$ \\
\hline Número de consultas (por semestre) & 185 & 154 & 211 & 136 \\
Número de pacientes de primera consulta & 381 & 345 & 397 & 291 \\
Número de pacientes de consultas sucesivas & 1.501 & 1.260 & 1.434 & 746 \\
Número total pacientes ambulatorios & 1.882 & 1605 & 1.831 & 1.037 \\
Número total pacientes ingresados & 710 & 816 & 794 & 944 \\
Número de quirófanos & 26 & 15 & 20 & 7 \\
Número pacientes (técnicas invasivas & 78 & 62 & 89 & 33 \\
realizadas en quirófano) & 211 & 169 & 231 & 143 \\
Número jornada de anestesiólogos & 2.592 & 2.421 & 2.625 & 1.981 \\
Total pacientes & & & & 7 \\
\hline
\end{tabular}

1S: primer semestre. 2S: segundo semestre. Total de pacientes se obtiene por la suma de pacientes atendidos en régimen ambulatorio y de aquellos ingresados en el hospital.

en la práctica médica entre los pacientes en función de sus recursos económicos. En nuestro medio, las medidas políticas puestas en marcha generan dificultades en el acceso a los recursos sanitarios por parte de los pacientes, sobre todo de aquellos que pertenecen a grupos socioeconómicos menos favorecidos, provocando diferencias en los resultados médicos asistenciales. Estos resultados se hallan en sintonía con los postulados por Tamblyn y cols. (10) que centra en los pacientes con enfermedades crónicas los cambios más pronunciados en las nuevas políticas sanitarias tipo copago.

Un incremento del coste de los fármacos para las personas de edad avanzada puede llevar a reducciones en el uso 
de fármacos esenciales, junto a un aumento de la tasa de acontecimientos adversos y el consiguiente aumento global del gasto sanitario (10). Resulta llamativo que no son los pensionistas los que se ven más afectados por esta situación de crisis, sino que principalmente fueron desempleados con o sin prestaciones, población inmigrante y pacientes con un bajo nivel de estudios los que presentaron una peor situación económica. La falta de acceso sanitario de las personas inmigrantes puede reforzar los riesgos para la salud asociados a las condiciones socioeconómicas desfavorables $(11,12)$. Existen evidencias científicas del impacto negativo sobre la salud por el desempleo y por la precariedad laboral $(13,14)$ y así la Organización Mundial de la Salud señala al trabajo y empleo como expresión del trabajo remunerado, como uno de los principales determinantes sociales de la salud (15).

Al contrario que otros trabajos (16), no hallamos relación entre sexo y desigualdades económicas, quizás por la existencia de un antiguo estado de bienestar o quizás por el gran impacto y duración de la actual crisis económica.

La necesidad de cumplir con los compromisos europeos relativos a déficit y deuda desencadenan recortes en protección social, solvencia y equidad de nuestro estado de bienestar (17). El cambio en las políticas farmacéuticas a nivel europeo es un hecho constatable (8) y entre las medidas desarrolladas para la contención del gasto sanitario se hallan el copago sanitario, incremento de un pago ya existente, o su ampliación a personas que deberían pagar (9). Una de las opciones planteadas en España en este periodo de crisis ha sido la exclusión de determinados fármacos y el incremento del copago de otros. Estas políticas sanitarias han podido determinar que en nuestro trabajo aquellos pacientes que presentan una peor situación económica redujeran el número de analgésicos empleados para el tratamiento de su patología, e incluso, rechazaron diversas opciones farmacológicas. Estos resultados ponen de manifiesto que la reducción de recursos destinados a sanidad y el aumento del coste para los pacientes provocan efectos negativos sobre la equidad, concordando nuestros estudios con otros ya publicados $(9,10)$. Existen grupos de presión influyentes interesados en el consumo de novedades farmacéuticas y en este punto debemos enfatizar la importancia del personal médico, el cual debe ser consciente a la hora de la prescripción de medicamentos del notable incremento del coste de las diversas novedades terapéuticas para el paciente y el propio sistema de salud. En este sentido, es de reseñar los hallazgos encontrados entre el nivel de recursos económicos escasos con la prescripción del número de fármacos, novedades terapéuticas y el empleo de técnicas intervencionistas como primera opción de tratamiento. Esta modulación del tratamiento en función de la renta se asocia con un incremento de los costes hospitalarios y de las listas de esperas quirúrgicas, por lo que sería interesante posteriores estudios de análisis de resultados.

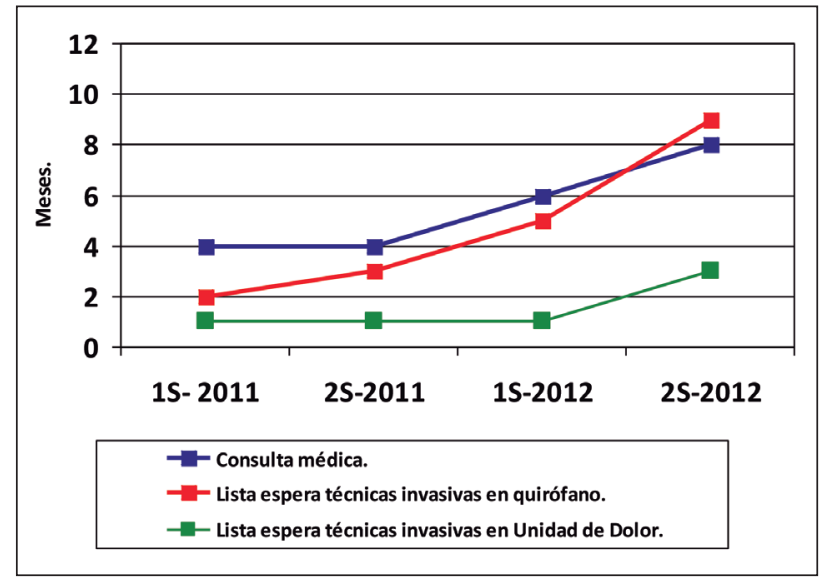

Fig. 2. Evolución de la lista de espera de primera consulta y lista de espera quirúrgica de Unidad de Dolor (1S: primer semestre, 2S: segundo semestre) por años de estudio.

Los datos de nuestro estudio exponen un progresivo aumento de las listas de espera, tanto para primera consulta en la Unidad del Dolor, como para la realización de procedimientos intervencionistas. Las circunstancias que rodean a este deterioro de la asistencia fueron multifactoriales, como es un progresivo aumento de la demanda asistencial, la reducción del número de quirófanos de otras especialidades quirúrgicas, de consultas médicas, etc.

Otra cuestión que debemos tratar es la relación nivel de estudios-situación económica. Los beneficios de la educación sobre la salud constituyen uno de los denominados beneficios no monetarios de la educación $(18,19)$. A mayor nivel de estudios, la situación económica suele ser más favorable, por lo que en este punto es necesario hacer también una reflexión en cuanto a la reducción en los recursos destinados a educación. Es conocido que las inversiones educativas en la primera infancia son eficientes a largo plazo también en términos de salud (20). Si tenemos una sociedad peor formada y el nivel de estudios desciende, esto conllevará una peor situación económica personal y/o familiar. En nuestro caso la precariedad económica se correlaciona de forma significativa con una mayor incidencia de trastornos en la esfera mental.

Las limitaciones del nuestro estudio son varias. Por un lado, el periodo de estudio, ya que se limita a los dos últimos años y no podemos observar la correlación desde el inicio de la crisis. En segundo lugar, la provincia de Almería muestra unas tasas de población inmigrante elevadas, por lo que los datos del presente estudio podrían ser no extrapolables a otras zonas geográficas de nuestro país. En tercer lugar, la aprobación del Real Decreto-Ley 16/2012, de 20 de abril, de medidas urgentes para garantizar la sostenibilidad del Sistema Nacional de Salud y mejorar la calidad y seguridad de sus prestaciones (21) ha podido conllevar una peor situación que la reflejada en nuestro 
trabajo. Deberían realizarse investigaciones multicéntricas para conocer si la repercusión de la crisis económica influye de la misma manera en toda la geografía española.

En conclusión, las crisis económicas ofrecen oportunidades para el cambio al facilitar modificaciones organizativas y son una excelente oportunidad para racionalizar los sistemas de salud buscando la rentabilidad social de las políticas $(22,23)$. Es importante una definición de catálogos de prestaciones basados en criterios de coste-efectividad y un cambio de las condiciones de financiación mediante políticas de genéricos, rediseño racional de los copagos, contratos de riesgo compartidos con la industria, mayor unión entre atención primaria y especializada, etc. En segundo lugar, el crecimiento de la desigualdad puede estar en el origen de la crisis económica y esta, a su vez, genera nuevos niveles de desigualdad y brechas en la equidad social y sanitaria agravando la crisis social. Es fundamental una mejor gestión de los recursos disponibles, de manera que los ajustes no favorezcan la aparición de nuevas desigualdades. Se deben incorporar en los diferentes sistemas de salud evaluaciones periódicas del impacto en términos de salud de estas políticas de austeridad.

\section{CORRESPONDENCIA}

Manuel Cortiñas Sáenz

Servicio de Anestesiología y Reanimación

Hospital Torrecárdenas

c/ Hermandad de Donantes de Sangre, s/n

04009 Almería

e-mail: mcortinassaenz@gmail.com

\section{BIBLIOGRAFÍA}

1. Quaglio G, Karapiperis T, Van Woensel L, et al. Austerity and health in Europe. Health Policy 2013;113:13-9.

2. McKee M, Karanikolos M, Belcher P, et al. A failed experiment on the people of Europe. Clinical Medicine 2012;12:346-50.

3. Kondilis E, Giannakopoulos S, Gavana M, et al. Economic crisis, restrictive policies, and the population's health and health care: the Greek case. Am J Public Health 2013; 103:973-9.

4. Liaropoulos L. Greek economic crisis: Not a tragedy for health. BMJ 2012;345:e7988.

5. González B. La sanidad en tiempos de crisis. España 2010. Un balance. Revista Economistas 2011;126:127-34.

6. Dávila C, González B. Crisis económica y salud. Gac Sanit 2009;23:261-5.

7. Consejería de Salud, Junta de Andalucía. Plan andaluz de atención a las personas con dolor: 2010 - 2013. Disponible en: http://www.juntadeandalucia.es/salud/export/sites/ csalud/galerias/documentos/c_1_c_6_planes_estrategias/ plan_de_atencion_al_dolor/plan_atencion_dolor.pdf.

8. Regidor E, Barrio G, Bravo MJ, et al. Has health in Spain been declining since the economic crisis? J Epidemiol Community Health 2013 Oct 23. doi: 10.1136/jech-2013202944.

9. Sydenstricker E. Health in the new deal. Annals of the American Academy of Political and Social Science 1934; 176:131-7.

10. Tamblyn R, Laprise R, Hanley JA, et al. Adverse events associated with prescription drug cost-sharing among poor and elderly persons. JAMA 2001;285:421-9.

11. Chauvin P, Parizot I, Simonnot N. El acceso a la sanidad de las personas sin permiso de residencia en once países europeos. Informe del Observatorio Europeo - Médicos del Mundo; 2009.

12. López-Fernándeza L, Martínez JI, Fernández A, et al. ¿Está en peligro la cobertura universal en nuestro Sistema Nacional de Salud? Gac Sanit 2012;26:298-300.

13. Artazcoz L, Benach J, Borrell C, et al. Unemployment and mental health: Understanding the interactions among gender, family roles, and social class. Am J Public Health 2004;94:82-8.

14. Benavides FG, Benach J, Diez-Roux AV, et al. How do types of employment relate to health indicators? Findings from the Second European Survey on Working Conditions. J Epidem Community Health 2000;54:494-01.

15. Comisión de Determinantes Sociales de la Salud. Subsanar las desigualdades sociales en salud en una generación. Ginebra: Organización Mundial de la Salud. 2009. Disponible en: http://whqlibdoc.who.int/publications/2009/ 9789243563701 spa.pdf.

16. Stephen G, Roberts A. Macroeconomic governance, gendered inequality, and global crises. En: Young B, Bakker I, Elson D, editors. Questioning financial governance from a feminist perspective. Routledge; 2011. p. 155-70.

17. Urbanos R. Desigualdades sociales en salud: malos tiempos para la lírica. Gac Sanit 2012;26:101-2.

18. Groot W, Maassen van den Brink H. The health effects of education. Economics of Education Review 2007;26:186-200.

19. Groosman M, Kaestner R. Effects of education on health. En: Behrman J, Stacey N, editors. The social benefits of education. Ann Arbor: University of Michigan Press; 1997. p. 69-123.

20. Cutler D, Lleras-Muney A. Education and health: Insights from international comparisons. NBER Working Papers W17738. 2012;1:30.

21. Real Decreto-Ley 16/2012, de 20 de abril, de medidas urgentes para garantizar la sostenibilidad del Sistema Nacional de Salud y mejorar la calidad y seguridad de sus prestaciones. BOE n. ${ }^{\circ}$ 98, 24 de abril de 2012; Sec. I: 31278-312.

22. García-Sánchez I, Pinilla Muñoz J, Escudero Espinosa C, et al. Guía de buenas prácticas para mejorar la facturación por la asistencia sanitaria a ciudadanos de la UE en el SSPA. Granada: Observatorio de Salud en Europa de la Escuela Andaluza de Salud Pública; 2011.

23. Repullo JR, Freire JM. Gobernabilidad del Sistema Nacional de Salud: mejorando el balance entre los beneficios y los costes de la descentralización. Informe SESPAS 2008. Gac Sanit 2008;22:118-25. 